\title{
Rapid diversification of homothorax expression patterns after gene duplication in spiders
}

\author{
Natascha Turetzek ${ }^{1,2,4}$, Sara Khadjeh ${ }^{1,2,3}$, Christoph Schomburg ${ }^{1,2}$ and Nikola-Michael Prpic ${ }^{1,2^{*}}$ (D)
}

\begin{abstract}
Background: Gene duplications provide genetic material for the evolution of new morphological and physiological features. One copy can preserve the original gene functions while the second copy may evolve new functions (neofunctionalisation). Gene duplications may thus provide new genes involved in evolutionary novelties.

Results: We have studied the duplicated homeobox gene homothorax (hth) in the spider species Parasteatoda tepidariorum and Pholcus phalangioides and have compared these data with previously published data from additional spider species. We show that the expression pattern of hth 1 is highly conserved among spiders, consistent with the notion that this gene copy preserves the original $h$ th functions. By contrast, $h$ th 2 has a markedly different expression profile especially in the prosomal appendages. The pattern in the pedipalps and legs consists of several segmental rings, suggesting a possible role of hth2 in limb joint development. Intriguingly, however, the $h$ th2 pattern is much less conserved between the species than $h$ th 1 and shows a species specific pattern in each species investigated so far.

Conclusions: We hypothesise that the hth2 gene has gained a new patterning function after gene duplication, but has then undergone a second phase of diversification of its new role in the spider clade. The evolution of hth2 may thus provide an interesting example for a duplicated gene that has not only contributed to genetic diversity through neofunctionalisation, but beyond that has been able to escape evolutionary conservation after neofunctionalisation thus forming the basis for further genetic diversification.
\end{abstract}

Keywords: Gene duplication, Neofunctionalisation, Spider, Homothorax, Gene expression, Evolution

\section{Background}

Diversification of morphological traits during evolution also requires diversification at the molecular level. Apart from the emergence of entirely new genes [1-3], diversification of gene function can be achieved either through changes at the regulatory level or by alterations in the coding region of the gene. Most genes have more than one function (pleiotropy), thus changes in their coding region and to a lesser extent in their regulatory regions will not only change a single feature of its function, but

\footnotetext{
* Correspondence: nprpic@uni-goettingen.de

${ }^{1}$ Abteilung für Entwicklungsbiologie, Johann-Friedrich-Blumenbach-Institut für Zoologie und Anthropologie, Georg-August-Universität, Göttingen, Germany

${ }^{2}$ Göttingen Center for Molecular Biosciences (GZMB), Ernst-Caspari-Haus, Göttingen, Germany

Full list of author information is available at the end of the article
}

usually cause multiple (and mostly negative) effects in different organs or tissues (reviewed in [4, 5]). One possibility for overcoming this problem and facilitating evolutionary changes in pleiotropic genes is gene duplication: one copy preserves the original functions of the pleiotropic gene and thus allows the second copy to evolve in an (at least theoretically) almost unrestricted fashion.

Indeed, there is an increasing number of studies that provide evidence for a prominent role of gene duplication in the evolution of physiological or morphological novelty. Especially in plants where duplications of the entire genome are frequent, gene duplications have been implicated in the evolution of morphological novelties [6-8]. But also in animals, gene duplications have been linked to evolutionary novelties, for example in the evolution of spermatogenesis, the immune system, protective embryonic membranes, the physiology of digestion, 
and the morphology of the exoskeleton in Drosophila species $[9,10]$, sexual dimorphism in stalk-eyed flies [11], the visual system in crustaceans and insects [12], sex determination in bees [13], electric organs and the bulbus arteriosus in fish [14, 15], or the silk producing apparatus and leg morphology in spiders $[16,17]$.

In the classical model of gene duplication ([18], discussed in $[19,20])$, the new copy evolves fast, because it is redundant and therefore is not subject to selection. It thus diverges quickly from the other copy which remains evolutionarily conserved, because of stabilizing selection on the old function. However, if the new copy becomes essential either by subfunctionalisation or neofunctionalisation, it is no longer redundant and from that time point it is also evolutionarily conserved because of its new functionality. The typical outcome of this process is that duplicated genes within a species (paralogs) differ in their expression profile, but the expression pattern of each paralog is evolutionarily conserved between different species.

The genomes of arachnids (including spiders) contain a large number of duplicated genes, and it has already been suggested that these may have fueled the evolution of many arachnid-specific novelties [16, 21, 22]. Previous work has shown that the homothorax (hth) gene is duplicated in the spider species Cupiennius salei (C. salei) and Acanthoscurria geniculata (A. geniculata) [23, 24]. The $h$ th genes are widely conserved in the Metazoa and homologs have been described from all major arthropod clades [25], onychophorans [26], and from vertebrates, where the gene is called Meis [27]. The hth gene of Drosophila melanogaster (D. melanogaster) codes for a transcription factor with two highly conserved protein domains. The MEIS domain is a protein-protein binding domain that mediates the binding between $\mathrm{Hth}$ and a co-factor named Extradenticle (Exd) [28-30]. In addition to the MEIS domain the Hth protein also contains a modified homeodomain (TALE-HD) that facilitates the binding of Hth to DNA (e.g. [28]). In many cellular contexts Hth first binds to Exd in the cytoplasm, and the protein pair is then translocated to the nucleus where they bind DNA together with Hox proteins [31-35]. Apart from Exd and Hox proteins, Hth is also known to bind other DNA binding proteins (e.g. [36]). Therefore, $h$ th is a highly pleiotropic gene and is involved in the formation and function of a large number of organs and tissues, for example leg and antenna specification, eye development, renal tubule growth, muscle fiber identity, and regulation of neuron differentiation (e.g. [31, 37-41]).

The hth duplicates in the spiders C. salei and A. geniculata show a strikingly different expression pattern, especially in the legs. One paralog, termed hthl, is expressed in a uniform pattern in the proximal and medial parts of the legs in both species [23, 24, 42]. This expression pattern is similar to hth expression in other arthropods and therefore likely is the conserved original expression of the gene before gene duplication [23]. The second paralog, termed $h t h 2$, is expressed in repeated rings along the proximal-distal axis of the legs in both species. This patterning is not seen in hth genes from other animals, and therefore likely represents a novel patterning function after divergence and neofunctionalisation of this second duplicate [23]. Therefore, at first glance, the duplicated $h t h$ genes in spiders show the typical behaviour of duplicated genes after neofunctionalisation: divergence of expression patterns between the paralogous genes, but strong conservation of the old and the new expression patterns between species. However, the ring pattern of $h t h 2$ is not entirely identical in $C$. salei and A. geniculata as noted by Pechmann et al. [43]. We have studied $h t h$ genes from two additional spider species and show that the expression patterns of $h t h 2$ indeed differ quite substantially between the species. We hypothesise that the $h t h 2$ gene has gained a new patterning function after gene duplication, but has then undergone a second phase of diversification of its new role. The $h t h 2$ gene is apparently a spider specific duplication and not related to other $h$ th duplication events of nonspider arachnids. Diversification of $h$ th 2 expression patterns would thus be an interesting example for a duplicated gene that has not only provided genetic diversity through neofunctionalisation, but beyond that has been able to escape evolutionary conservation after neofunctionalisation thus becoming the basis for further genetic diversification.

\section{Methods}

\section{Animal cultivation and gene cloning}

Parasteatoda tepidariorum (P. tepidariorum) and Pholcus phalangioides (Ph. phalangioides) embryos were obtained from our laboratory stocks in Göttingen, and were treated as described previously $[17,44]$. For gene cloning, we used SMARTer RACE cDNA (Clontech, Mountain View, CA, USA) synthesized from a combination of all embryonic stages of the different spider species (for details see [17]). Initial fragments of $P p$-hth1 and Pt-hth2 were amplified using nested PCR with degenerated primers as described in [23]. Larger gene fragments for $P p$-hth1 were subsequently obtained by RACE PCR using the following primers $P p-h t h-3 R A C E$ : GGA CAT CCA TTG TTT CCA TTG TTG G, Pp3RaceHthnew: CAG GGA CTG CGA CGG GGG CCT C. Pp$h t h 2$ was cloned with the following gene specific primers that were designed based on sequence information of a preliminary de novo assembled $P h$. phalangioides transcriptome (unpublished data):

Pp_hth2_for: CGGTTATCGGGTGGACTTCGG.

Pp_hth2_rev: GGTCCATGATGTTCGGAGGCGAA. 


\section{Phylogenetic analysis}

For the phylogenetic analysis of $h t h$ we searched for larger/complete open reading frames in the transcriptomes of Ph. phalangioides (unpublished data) and P. tepidariorum [45] using D. melanogaster and C. salei hth homologs as search queries. With the same query sequences we also searched in the published genome resource of the scorpion Mesobuthus martensii (M. martensii) [21], which resulted in three fragments with similarity to $h$ th, named MMa38611, MMa00258, and MMa39038 (note that MMa00258 is very short and thus might be an incompletely assembled fragment). For the protein and nucleotide alignments of the $h t h$ orthologs we used previously published $h$ th-related sequences from various metazoan species plus all $h$ th sequences identified in the transcriptomes of P. tepidariorum and Ph. phalangioides and the genome of $M$. martensii (see list of species and accession numbers in Additional file 1).

Sequences were aligned with Clustal Omega [46] using default settings (the alignments are available in Nexus format in Additional file 2 and Additional file 3). Phylogenetic trees were calculated using the parallel version of MRBAYES (version 3.2.5) [47]. For the protein alignment, after testing mixed amino acid substitution models the Jones model was chosen to generate topological convergence using Metropolis coupling for 179,000 generations after reaching an average standard deviation of split frequencies below 0.01. A total number of 718 trees were written in 2 files (each file contained 359 trees of which 270 were sampled). In the nucleotide sequence analysis (using Metropolis coupling and the 4by4 nucleotide substitution model) an average standard deviation of split frequencies of 0.023742 was reached after 9 million generations. A total of 36,002 trees were written in 2 files $(18,001$ trees per file of which 13,501 were sampled). The $50 \%$ majority rule consensus trees shown in Fig. 1 and Additional file 4 were visualized with Geneious (version 9.1.3) [48].

\section{Whole mount in situ hybridization and imaging}

Fixation and in situ hybridization of embryos were performed according to standard protocols with minor modifications [49]. Digoxygenin-labeled RNA probes were synthesized from RACE PCR fragments for $P p-h t h 1$, the entire $P p$-hth 2 and $P t-h t h 2$ fragments, and from the previously published fragment of Pt-hth1 (GenBank accession number HE608682 [50]). The location of the probes is indicated in Additional file 5. This shows that the probes overlap highly conserved regions and thus in principle could produce unwanted cross-hybridization. However, given the distinct expression patterns detected with the used probes, we think that cross-hybridization was minimal. After in situ hybridization and cell nuclei staining with SYTOX-Green (Invitrogen), whole embryos were imaged with a Leica M205 FA binocular equipped with a QImaging Micropublisher 5.0 RTV camera using combined UV and white light. In addition appendages were dissected and mounted in 80\% Glycerol in PBST and images were captured with a Zeiss Axioplan-2 microscope equipped with an Intas digital camera. For colour and brightness correction of all images Adobe Photoshop CS5 Extended or CS6 for Apple Macintosh was used. Staging of embryos was performed on the basis of the published

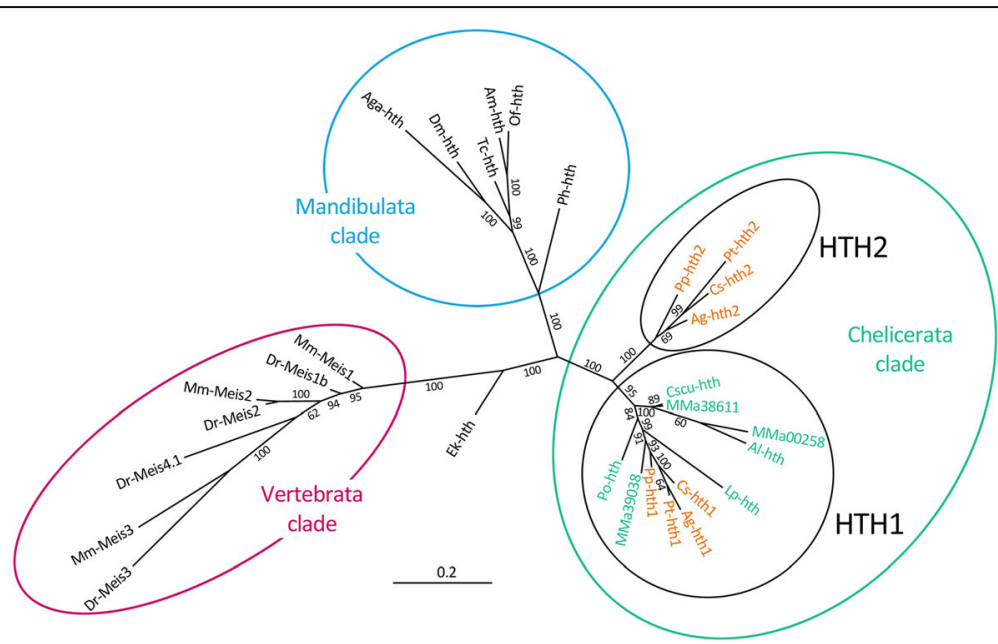

Fig. 1 Phylogenetic analysis of $h$ th and related protein sequences from diverse Metazoa. Unrooted 50\% majority rule consensus tree after Bayesian Markov chain Monte Carlo analysis. Branch lengths in the phylogram give the expected substitutions per site. Numbers at the tree edges are clade credibility values, which are a measure of the probability of each clade in the tree. The monophyletic clades formed by all Meis sequences from vertebrates, the hth sequences from mandibulate arthropods, and those from chelicerates are indicated by coloured circles (red, blue and green, respectively), Within the Chelicerata clade $h$ th sequences from non-spider chelicerates (horse-shoe crab, scorpions, harvestman and mite) are indicated in green, whereas spider sequences are highlighted in orange). Homologs of spider hth 1 and hth2 are indicated by black circles. For species abbreviations and sequence accession numbers please see Additional file 1 
staging schemes for P. tepidariorum [51], C. salei [52], and Ph. phalangioides [44].

\section{Results \\ Duplicated hth genes in P. tepidariorum and $P h$. phalangioides}

Previous studies in the entelegyne spider C. salei [23] and the bird spider A. geniculata [25] have shown that $h t h$ is duplicated in these species, and that the genes apparently form two separate phylogenetic groups, termed $h t h 1$ and $h t h 2$, that differ in spatio-temporal expression. To investigate whether the duplication of $h t h$ is a general feature present in all spider groups, we have studied two additional spider species that belong to two remotely related spider groups, the cellar spider $P h$. phalangioides and the common house spider P. tepidariorum, representing a haplogyne and another entelegyne spider, respectively. At the beginning of the present study transcriptome resources were not available for these species, therefore hth-related sequences were amplified from cDNA preparations with degenerated primers and more sequence information was obtained via RACE-PCR (see Methods). As expected from previous studies in spiders we were able to isolate partial sequences of two hth paralogs in $P$. tepidariorum. For Ph. phalangioides we only identified the hth1 homolog. When transcriptome resources became available, we also searched for $h t h$-related sequences in the now available developmental transcriptomes of $P$. tepidariorum [45] and Ph. phalangioides (unpublished data). These sequencing resources confirmed the presence of two hth homologs in P. tepidariorum, and also revealed the presence of a second hth homolog in Ph. phalangioides that had escaped previous conventional gene cloning attempts. The fact that duplicated $h t h$ genes are now known from representatives of most major spider lineages including entelegyne spiders, haplogyne spiders and mygalomorph spiders, suggests that this gene is duplicated in all spiders.

\section{Phylogenetic analysis reveals duplication of $h$ th early in spider evolution}

We next asked if these duplicates derive from a single duplication event before the radiation of the spider lineages, or if $h t h$ has been duplicated several times independently in the different spider groups. A previous phylogeny with all then available spider sequences had suggested that the duplicated $h t h$ genes cluster together according to duplicate and not according to species, suggesting a duplication before the split between mygalomorph and entelegyne spiders [24]. We therefore reconstructed the phylogeny of $h t h$ and related genes using published hth sequences from chelicerates, mandibulates, onychophorans, and the vertebrate Meis genes (see Additional file 1 for a full list of species and sequence accessions). Protein and nucleotide sequences were analysed separately and resulted in phylogenetic trees with very similar topology (Fig. 1, Additional file 4). The vertebrate Meis sequences form a monophyletic group in the trees and are clearly separated from the $h t h$ sequences from arthropods and the onychophoran. The insect and crustacean sequences also form a monophyletic group that is well separated from the group that contains the chelicerate sequences. Interestingly, the chelicerate sequences are divided into two separate monophyletic groups. The first group (denoted as hth1 in Fig. 1 and Additional file 4) contains all sequences from non-spider chelicerates (including three hth fragments (MMa38611, MMa00258, and MMa39038) found in the genome of the scorpion $M$. martensii, see Materials and Methods), the previously described $h$ th 1 copy from $C$. salei and A. geniculata, and one hth copy each from P. tepidariorum and Ph. phalangioides, that we therefore also denote as $h t h 1$. The second group (denoted as hth2 in Fig. 1 and Additional file 4) exclusively contains the hth 2 copy from C. salei and A. geniculata, and the second copy from $P$. tepidariorum and $P h$. phalangioides (therefore also denoted as hth2).

Expression of duplicated $h$ th genes during embryogenesis Our phylogenetic analysis suggests that the duplicated $h$ th genes in $P$. tepidariorum and $P h$. phalangioides are orthologs of the $h t h 1$ and $h$ th 2 genes previously described from $C$. salei and A. geniculata, and should therefore also show the diverged expression patterns in the legs. To test this assumption, we have studied the expression of the two hth genes in P. tepidariorum and Ph. phalangioides with a focus on the expression during embryonic leg development.

The expression of hth 1 is virtually identical in both species throughout embryonic development, especially in the prosoma. It is strongly expressed throughout the ventral neuroectoderm and in the anterior part of the head. During brain differentiation the expression in the head becomes restricted to the non-neurogenic ectoderm, the labrum, the stomodeum and two stripes next to the stomodeum (Figs. 2d and 3d). When the prosomal limb buds start developing, hth1 is expressed in the entire prosomal appendages excluding the most distal part (Figs. 2a and 3a). This strong expression in the prosomal appendages remains until late dorsal closure stages (Figs. 2b, c and 3b, c), but then decreases slightly, especially in the distal portion, mainly in the metatarsus (Figs. 4f and 5f). We found minimal differences in the opisthosomal expression patterns between the species. In P. tepidariorum, hth1 is expressed in the entire opisthosoma at uniform levels except for the posterior end and one spot next to the limb bud of the second opisthosomal segment (O2; Fig. 2e). In Ph. phalangioides the expression 


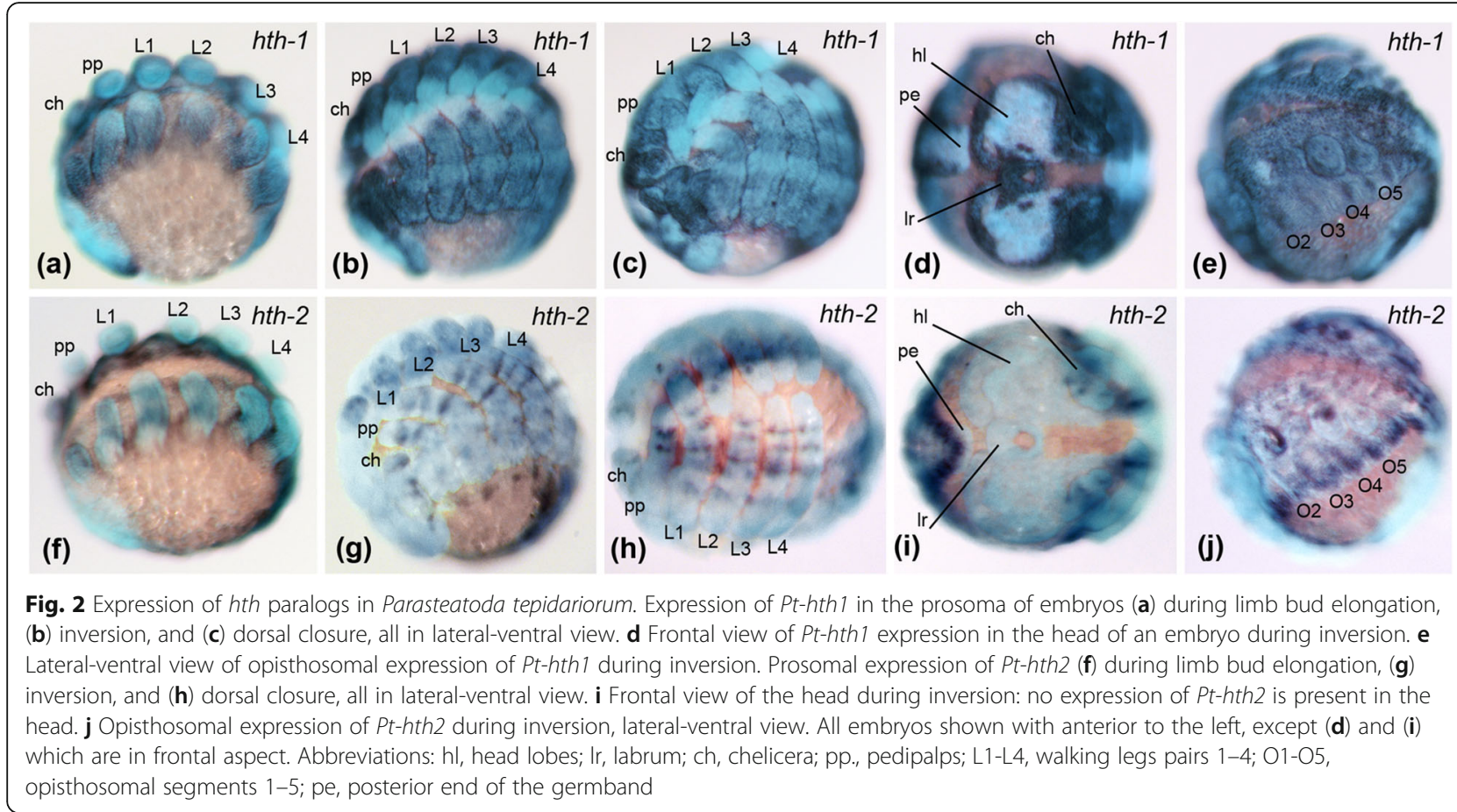

in the opisthosoma is more restricted to presumptive ventral tissue and no expression can be detected in the developing heart tissue (Fig. 3e).

Expression of $h$ th 2 differs from $h t h 1$ in terms of timing and pattern. In both species, the gene is expressed later and in a more restricted fashion than $h t h 1$. At germ band stages $h$ th 2 is expressed more ventrally than $h t h 1$ in the neuroectoderm and an expression domain in the head tissue or the developing appendages is absent (Figs. 2f, i and 3f, i). First expression of hth 2 in the prosomal appendages is present at early inversion stages, which then differentiates until ventral closure stages (see next chapter), but is always different from hth1 expression (Figs. 2g, h, and 3g, h). The expression of $h t h 2$ in the opisthosoma differs significantly between $P h$. phalangioides and P. tepidariorum. In P. tepidariorum hth2

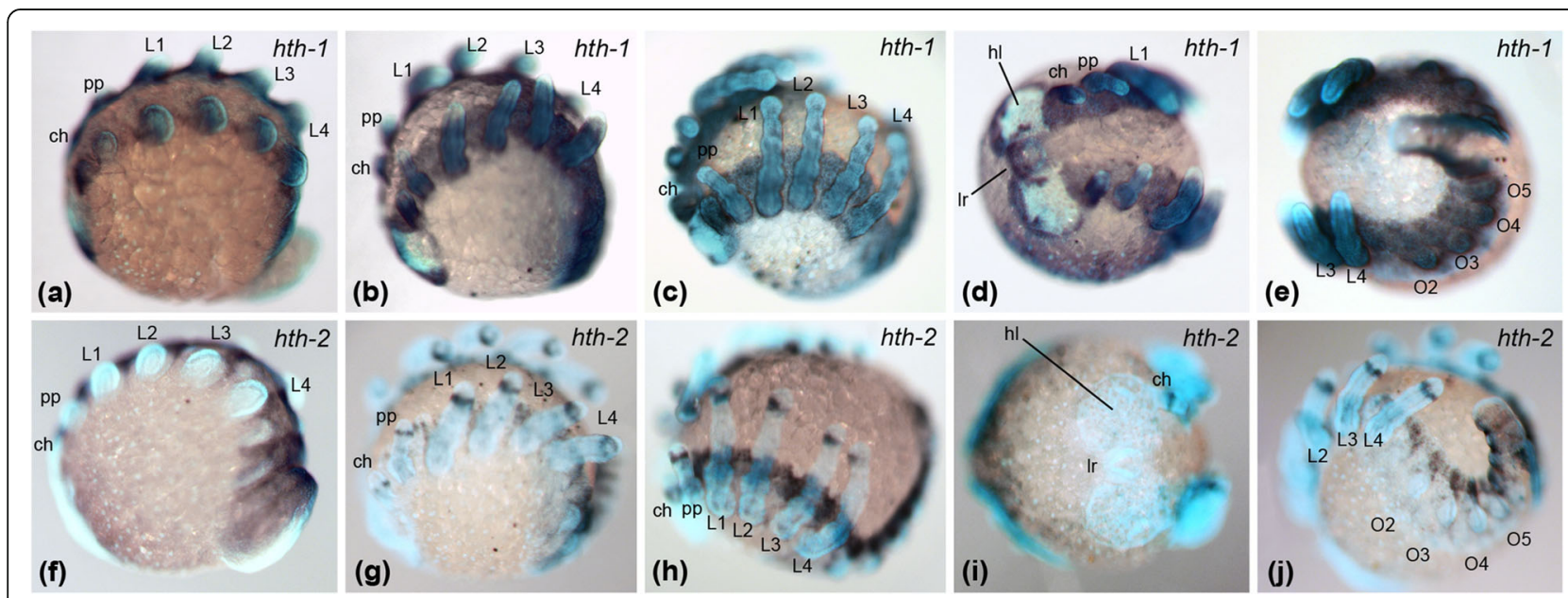

Fig. 3 Expression of hth paralogs in Pholcus phalangioides. Expression of Pp-hth1 in the prosoma of embryos (a) during limb bud elongation, (b) inversion, and (c) dorsal closure, all in lateral-ventral view. d Frontal view of $P$ p-hth1 expression in the head of an embryo during inversion. e Ventral view of opisthosomal expression of $P p$ - $h$ th1 during inversion. Prosomal expression of $P p$ - $h$ th2 $(\mathbf{f})$ during limb bud elongation, $(\mathbf{g})$ inversion, and (h) dorsal closure, all in lateral-ventral view. i Frontal view of the head during inversion: no expression of $P p$ - $h$ th2 is present in the head. $\mathbf{j}$ Opisthosomal expression of Pp-hth2 during inversion, lateral-ventral view. All embryos shown with anterior to the left, except (d) and (i) which are in frontal aspect. Abbreviations: see Fig. 2 


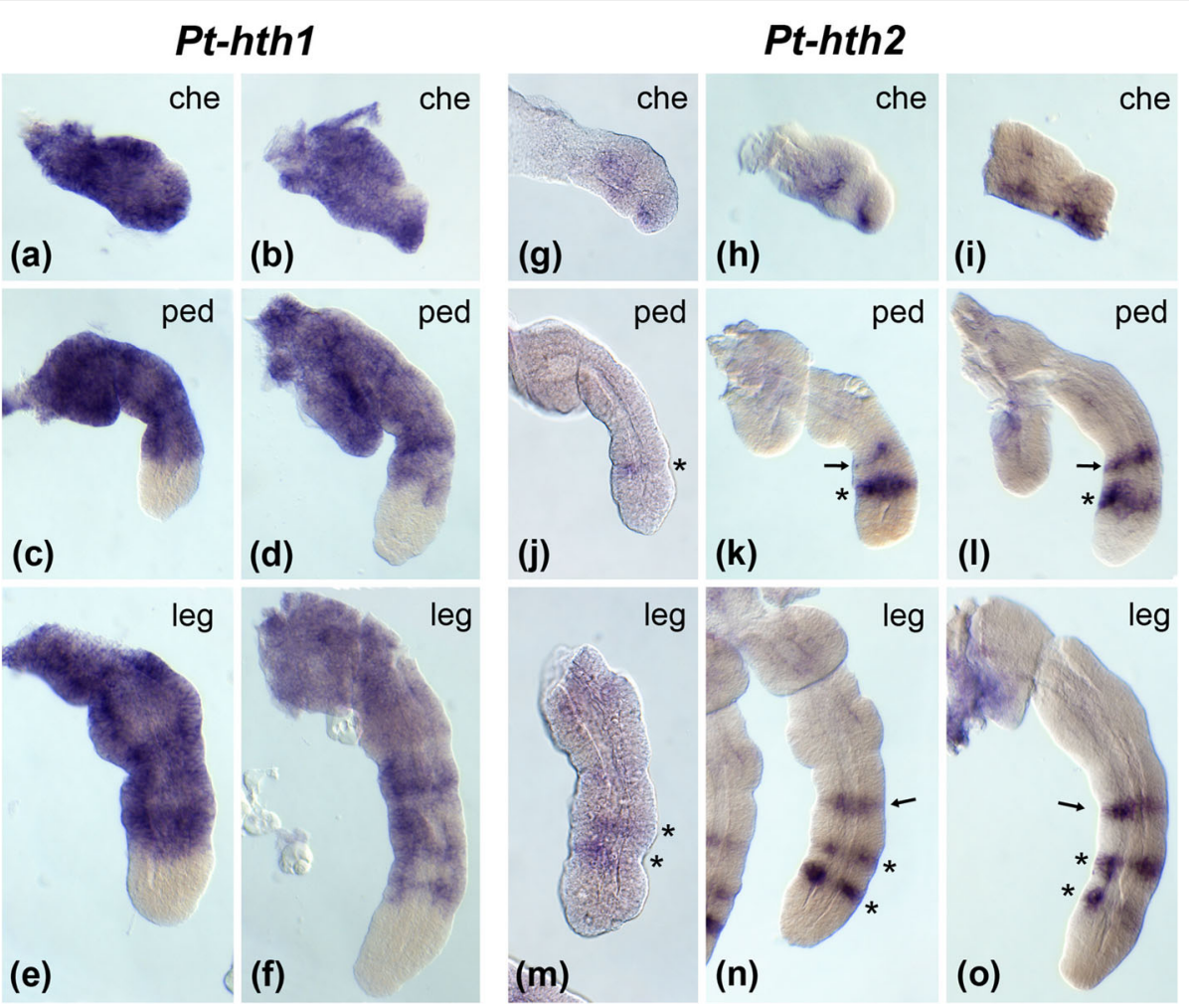

Fig. 4 Expression of hth paralogs in the prosomal appendages of Parasteatoda tepidariorum. a-f Expression of Pt-hth 1 is shown in the panels on the left side. g-o Expression of Pt-hth2 is shown in the panels on the right side in the figure. The top row of panels shows dissected chelicerae (che) $(\mathbf{a}, \mathbf{g})$ at early inversion, (h) late inversion, and $(\mathbf{b}, \mathbf{i})$ dorsal closure, the center row shows dissected pedipalps (ped) (c, $\mathbf{j})$ at early inversion, (k) late inversion and $(\mathbf{d}, \mathbf{I})$ dorsal closure, and the bottom row shows dissected walking legs $(\mathbf{e}, \mathbf{m})$ at early inversion, (n) late inversion, and $(\mathbf{f}, \mathbf{o})$ dorsal closure. The asterisks in (j-o) denote distal expression rings (one in the pedipalps, two in the leg). The arrows in (k, $\mathbf{l}, \mathbf{n}, \mathbf{o})$ denote an additional medial ring

is expressed adjacent to the opisthosomal limb buds, and dorsally in the tissue of the developing heart (Fig. 2j), but in Ph. phalangioides hth2 is not expressed in heart tissue and expression is restricted to the ventral side of the neuroectoderm and a spot ventral to each opisthosomal limb bud (Fig. 3j).

\section{Hth expression in the prosomal appendages of different spider species}

The largest expression differences between the hth duplicates exist in the prosomal appendages (chelicerae, pedipalps and walking legs). To analyse these differences in more detail we studied dissected prosomal appendages of P. tepidariorum and Ph. phalangioides. Expression of hth1 is very similar in P. tepidariorum and Ph. phalangioides. Strong expression of $h t h 1$ can be observed within almost the entire chelicera throughout embryonic development in both species (Figs. $4 \mathrm{a}, \mathrm{b}$ and $5 \mathrm{a}, \mathrm{b}$ ). Throughout most of development $h$ th 1 is also strongly expressed in the proximal and medial domain of the pedipalps and the walking legs, and is excluded from the tip of both appendage types. In embryos undergoing dorsal closure the medial expression becomes weaker and patchy (Figs. $4 \mathrm{c}-\mathrm{f}$ and $5 \mathrm{c}-\mathrm{f}$ ).
Expression of $h t h 2$ is quite different from the pattern of $h$ th 1 and also differs more significantly between the species. In the chelicera of $P$. tepidariorum hth 2 expression starts as a diffuse domain (Fig. $4 \mathrm{~g}$ ), that later forms two stripes or patches (Fig. 4h, i). In the pedipalps of $P$. tepidariorum hth 2 expression starts as a weak ring at the distal end of the medial portion of the pedipalp (asterisk Fig. 4j). A second ring appears later in development (arrow in Fig. 4k). In the legs Pt-hth2 is activated as two weakly expressed rings (asterisks Fig. 4m). An additional ring appears proximal to the initial two rings later in development (Fig. 4n, o (arrows)).

In Ph. phalangioides the hth 2 expression pattern also comprises ring-shaped domains, but they differ in number and expression dynamics from hth 2 in P. tepidariorum. In the chelicera $P p$-hth2 is first expressed as a ring in the medial part, which partially dissolves during development (Fig. 5g,h). In the pedipalp Pp-hth2 expression starts as one ring in the distal part, and a number of weak segmental rings appear more proximally later in development (Fig. 5i, j). In the walking legs $P p$ - $h$ th 2 expression also starts as a ring in the distal part (Fig. 5k) and later during development additional 


\section{Pp-hth1}

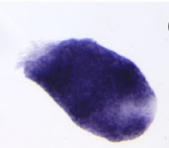

(a)

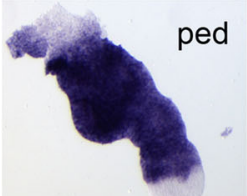

(c)

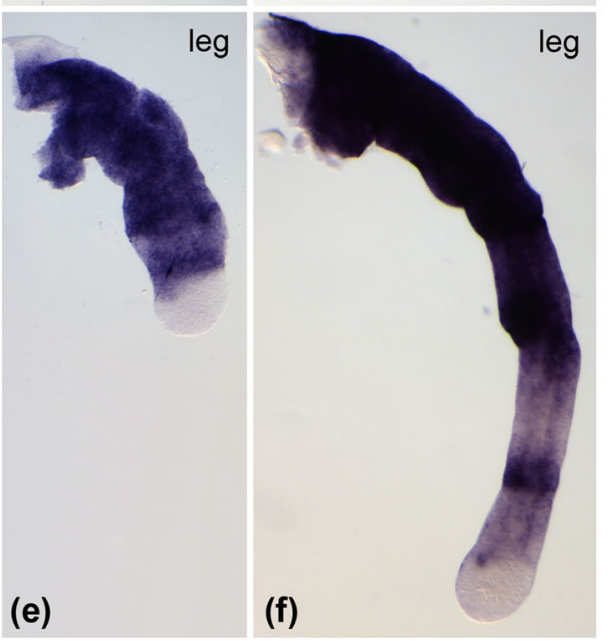

che
Pp-hth2

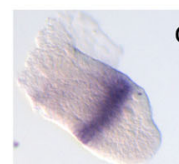

(g)

che

(h)
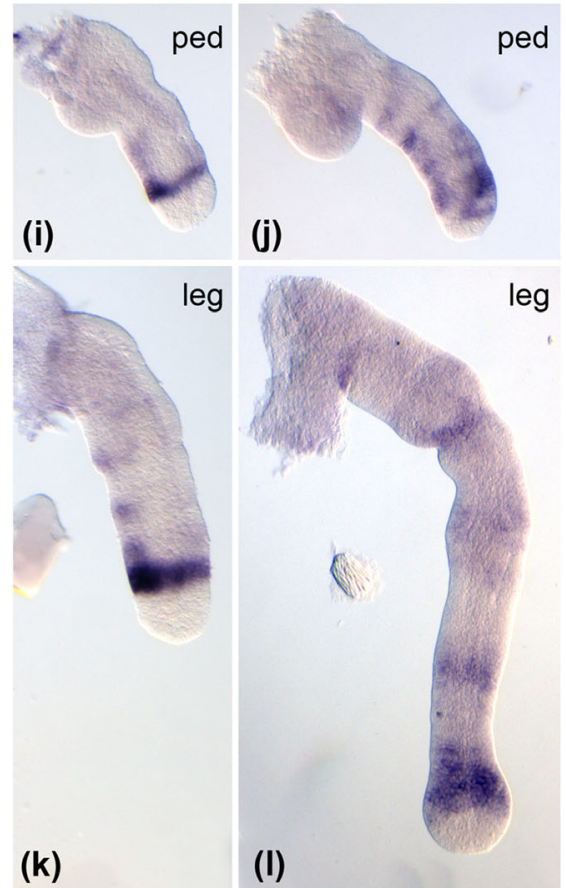

Fig. 5 Expression of hth paralogs in the prosomal appendages of Pholcus phalangioides. a-f Expression of Pp-hth1 is shown in the panels on the left side. $\mathbf{g}$-I Expression of $P p$-hth2 is shown in the panels on the right side in the figure. The top row of panels shows dissected chelicerae (che) $(\mathbf{a}, \mathbf{g})$ at inversion, and $(\mathbf{b}, \mathbf{h})$ shortly before ventral closure, the center row shows dissected pedipalps (ped) $(\mathbf{c}, \mathbf{i})$ at inversion, and $(\mathbf{d}, \mathbf{j})$ shortly before ventral closure, and the bottom row shows dissected walking legs $(\mathbf{e}, \mathbf{k})$ at inversion, and $(\mathbf{f}, \mathbf{I})$ shortly before ventral closure

weakly expressed rings appear near the future leg joints (Fig. 5l).

We have also re-studied here the expression profile of hth2 in C. salei, because in the original publication, only the expression pattern in the fully formed embryonic leg was shown [23], but the expression profile of this gene is actually more dynamic. Cs-hth2 has a diffuse expression in the chelicera with a faint medial ring (Fig. 6a). In young pedipalps it is weakly expressed in two rings (arrowhead and arrow, Fig. 6b). Later the proximal of these two rings broadens (arrow, Fig. 6c, d), the medial ring divides (arrowhead(s), Fig. 6c, d), and an additional distal ring appears de novo (asterisk, Fig. 6c, d). In young walking legs $C s-h t h 2$ is expressed in a proximal, medial and distal ring (arrow, arrowhead and asterisk, respectively, Fig. 6e). All three rings broaden during development (indicated by bars in Fig. 6f, g), and then split into two rings (Fig. 6h). This results in the presence of one ring near each of the developing leg joints in late stages of embryonic development.

\section{Discussion}

The hth 2 gene has originated by a spider specific $h$ th duplication

Duplicated $h$ th-related genes exist in vertebrates, scorpions, and spiders. Our phylogenetic analysis indicates that these gene copies do not trace from a single duplication event at the base of the metazoan phylogenetic tree, but have arisen in three independent gene duplication events (Fig. 1; Additional file 4). The ancestral state is the presence of a single $h t h$-related gene and this is preserved in the genomes of onychophorans and pancrustaceans. Also a number of chelicerate species have only a single copy of $h t h$, which clusters with the $h t h 1$ copy of spiders. This strongly suggests that the spider $h t h 1$ copy preserves the ancestral properties of $h t h$, which is also supported by the gene expression pattern (see also below).

Larger numbers of duplicated genes are found in several chelicerate groups including spiders, scorpions and horseshoe crabs $[16,21,22,53,54]$, but it is still unclear 


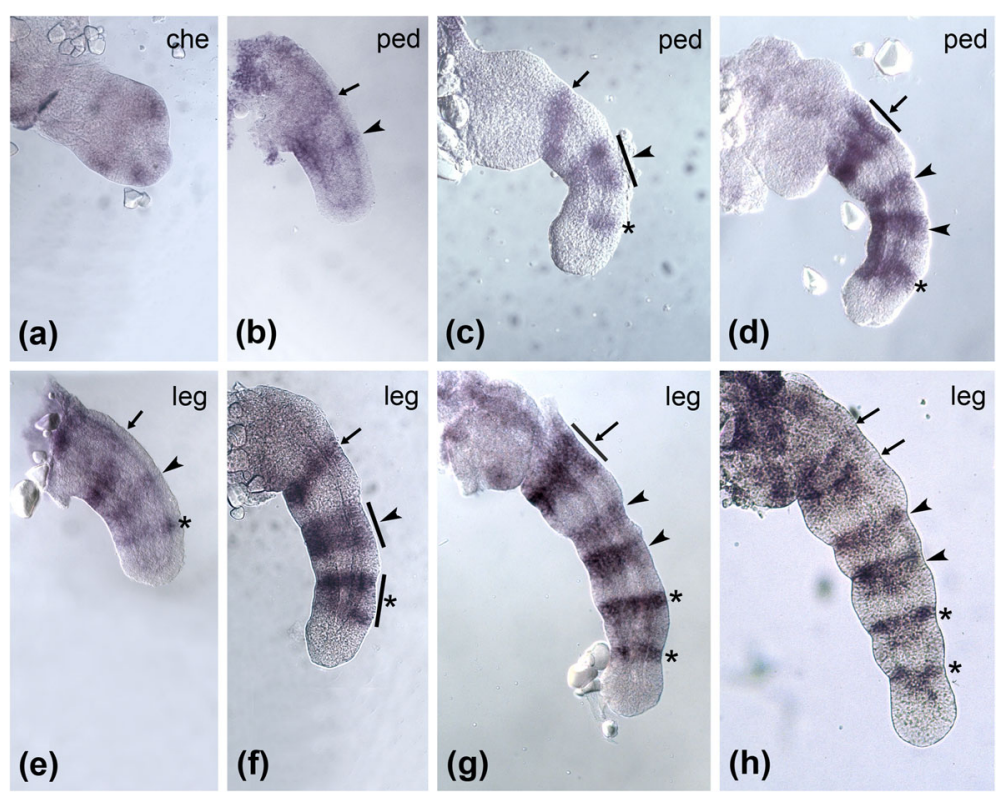

Fig. 6 Expression of hth2 in prosomal appendages of Cupiennius salei. a Weak expression of Cs-hth2 in the chelicera during late inversion. Expression of Cs-hth2 in the pedipalp (b) before inversion, (c) at early inversion and (d) at late inversion. The arrow points to the proximal Cs-hth2 ring which broadens during inversion (indicated by the bar). The arrowhead points to the medial Cs-hth2 ring, which broadens during inversion (bar in (c)) and splits at late inversion (two arrowheads in (d)). The asterisks mark the distal Cs-hth2 ring. Expression of Cs-hth2 in the walking legs (e) before inversion, (f) at early inversion, $(\mathbf{g})$ at late inversion, and (h) at dorsal closure. The arrows mark the proximal Cs-hth2 ring and its descendent. The arrowheads mark the splitting medial Cs-hth2 ring and the asterisks mark the splitting distal Cs-hth2 ring. Bars always indicate broadening of the ring expression before splitting

if these trace from a single large-scale gene or genome duplication event in the stem-line of the Chelicerata or if multiple gene duplications have occurred in several chelicerate lineages independently. We have previously shown that the duplication of the dachshund gene (dac) is an old duplication and has apparently occurred before the diversification of the arachnid orders [17]. Our phylogenetic analysis of $h t h$ shows that in contrast to $d a c$, the hth duplicates in scorpions and spiders are placed in separate clades in the phylogenetic tree. Thus, the $h$ th duplicates identified in spiders and scorpions are unlikely to trace from the same duplication event and therefore must have been duplicated independently after the split between scorpions and spiders. Genes of the hth 2 type are only found in spiders and therefore represent a relatively young $h$ th duplication event that is specific to spiders. The alternative hypothesis that $h t h$ has been duplicated at the base of the chelicerate or arachnid tree is less parsimonious, because a single $h t h$ gene is present in other chelicerate groups (including mites and harvestmen) and for these groups additional independent losses must then be assumed.

\section{Evidence for rapid diversification of $h$ th 2 regulation after gene duplication}

Our analysis of the gene expression patterns shows that the regulation of $h t h 1$ is highly conserved, resulting in virtually identical expression patterns in all four spider species. As noted previously [42], this highly conserved expression pattern is comparable with $h t h$ expression in other arthropods (e.g. [55-58]), onychophorans [26] and vertebrates (e.g. [59]). In addition, not only the regulation, but also the protein coding region of the $h$ th 1 genes is highly conserved: sequence alignments of the spider protein sequences show that the Hth1 amino acid sequences align with high overall identity (Additional file 6). This observation is further corroborated by the phylogenetic sequence analysis that shows that the spider Hth1 sequences group closely together, separated by few expected substitutions per site and thus are closely related phylogenetically (Fig. 1). Taken together, these data strongly suggest that the $h$ th 1 copy retained most of the original gene functions after gene duplication in spiders.

By contrast, the hth 2 copy has a markedly different expression profile in most tissues during embryonic development, especially in the prosomal appendages. The pattern in the pedipalps and legs consists of several segmental rings, suggestive of a role of $h t h 2$ in the development of the joints between the limb segments. Surprisingly this role is apparently not required in all segments of all species and each species investigated so far shows a unique pattern of segmental rings (summary in Fig. 7). This pattern does not correlate with the 


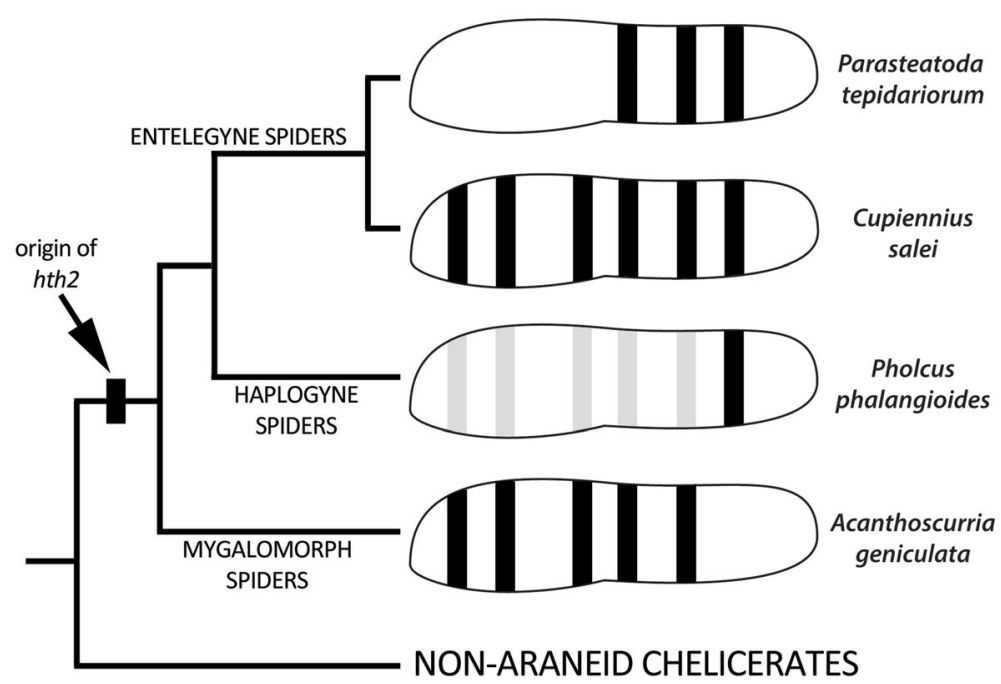

Fig. 7 Diversification of hth2 expression during spider evolution. The drawings show a schematic summary of hth2 expression in the walking legs in four different spider species (Acanthoscurria geniculata after [24]). Black stripes indicate strong expression in the walking legs, light gray bars represent weak expression. The cladogram at the left shows the phylogenetic relationships between the species (phylogeny simplified after [64]). The black box indicates the spider specific duplication event of hth that resulted in the origin of $h$ th 2 at the base of the spider phylogenetic tree

phylogenetic position of the species (e.g. the patterns in A. geniculata and C. salei are most similar, although both belong to distantly related clades in spider phylogeny). The pattern also does not correlate with the length of the adjacent segments and thus there is no evidence for a role in allometric limb growth. One possibility is that the divergent patters arose by neutral evolutionary processes, e.g. drift, that deleted/reduced different rings in different species. Alternatively, we suggest that expression/lack of expression of hth 2 at the joints might correlate with specific features at certain joints, but the comparative morphology of the limb joints in spiders has not been investigated in such detail to provide direct evidence for this hypothesis. Interestingly, most of the segmental rings of $h$ th 2 are located within the $h$ th 1 expression domain, i.e. hth 1 and the rings of $h$ th 2 (where present) are co-expressed at the limb joints. Thus, apart from a possible new function of hth 2 at the limb joints that would be independent of $h t h 1$, there is also the possibility that $h t h 1$ and $h t h 2$ act redundantly or cooperatively at selected limb joints to produce a certain morphology. As already mentioned in the introduction, the Hth protein usually binds to the Exd protein to be transferred to the nucleus; however, previous studies have shown that exd expression in $C$. salei is restricted to the proximal limb portion and a single distal ring [23]. Thus, most of the hth 2 rings in C. salei (and by inference also in the other species) are not co-expressed with exd and thus would require other means for their function. We have currently no further data on possible alternative binding factors for hth 2 in spiders, but we note that there is recent evidence that Hth can indeed function independently from Exd [60].

The gain of a novel role of a hth-related gene after gene duplication would not be unprecedented. Recent work in bats has shown that a duplicate of Meis, Meis2, is implicated in the growth of the wing membrane, a morphological novelty specific to bats [61]. However, the subsequent diversification of the expression pattern after the gain of the new function as seen in the expression patterns of $h t h 2$ in spiders is unusual. Normally genes are expected to conserve their new function after neofunctionalisation and therefore should not show rapid diversification after neofunctionalisation [18]. Our data suggest that genes can escape this pressure of conservation after neofunctionalisation, and thus further diversify. In addition, genuine hth 2 duplicates have so far only been found in opisthothelid spiders that originated in the Triassic period approximately 240 million years ago [62]. This makes the duplication event relatively young in terms of evolutionary age (relative to the age of the entire chelicerata clade which is at least 500 million years old [63]) and suggests that diversification of hth2 regulation must have occurred rapidly during spider radiation. Interestingly, also the Hth2 proteins (i.e. the coding sequence of the $h$ th 2 genes) from all four spider species studied so far are less similar among themselves than the Hth1 proteins. In sequence alignments the Hth2 amino acid sequences align with less overall similarity (Additional file 6 and Additional file 7), and in the phylogenetic analysis the spider Hth2 sequences are separated by more expected substitutions per site and thus are less closely related phylogentically than are the Hth1 
sequences. This suggests that after gene duplication subsequent diversification of $h t h 2$ not only affected its regulatory region, as evidenced by the diverse expression patterns, but to a certain extent also its protein coding region.

\section{Conclusions}

Our study of the duplicated homothorax genes in spiders shows that this gene duplication is specific to spiders and that the two copies differ significantly in their expression patterns. In addition, the expression pattern of the hth 2 copy is weakly conserved and shows a different expression pattern in each species studied so far. We conclude from these data that after duplication of $h t h$ at the base of the spider lineage the hth 1 copy preserved the original functions and therefore shows a pattern similar to the unduplicated $h t h$ gene in other arthropods. The $h t h 2$ gene on the other hand shows a strongly divergent expression pattern, likely indicating new functionality not shared with the hth 1 copy. We hypothesise that hth2 after neofunctionalization underwent a second phase of diversification of gene expression that led to the different expression patterns in each species. Thus, $h$ th 2 may serve as an interesting example for a duplicated gene that not only acquired new functionality, but also rapidly diversified this new functionality during evolution.

\section{Additional files}

Additional file 1: Species abbreviations and sequence sources used in the phylogenetic analyses. (DOCX $129 \mathrm{~kb})$

Additional file 2: Alignment of metazoan hth-related protein sequences used in the phylogenetic sequence analysis in Nexus format. (NEX 25 kb)

Additional file 3: Alignment of metazoan hth-related nucleotide sequences used in the phylogenetic sequence analysis in Nexus format. (NEX $63 \mathrm{~kb}$ )

Additional file 4: Phylogenetic analysis of hth and related nucleotide sequences from diverse Metazoa. Unrooted 50\% majority rule consensus tree after Bayesian Markov chain Monte Carlo analysis. Branch lengths in the phylogram give the expected substitutions per site. Numbers at the tree edges are clade credibility values, which are a measure of the probability of each clade in the tree. The monophyletic clades formed by all Meis sequences from vertebrates, the $h$ th sequences from mandibulate arthropods, and those from chelicerates are indicated in the figure. For species abbreviations and sequence accession numbers please see Additional file 1. (JPEG $548 \mathrm{~kb}$ )

Additional file 5: Location of the RNA probes indicated on the mRNA sequence of the individual $h$ th genes to indicate the overlap with conserved domains. The protein coding sequence (CDS) is shown in bold type, red colour indicates the location of the Meis domain, and blue colour indicates the location of the homeodomain. The location of the probe is mapped onto the sequence by gray background shading. (DOCX 206 kb)

Additional file 6: Alignment of Hth1 proteins from four spider species. Abbreviations: Pp, Pholcus phalangioides; Cs, Cupiennius salei; $\mathrm{Ag}$, Acanthoscurria geniculata; Pt, Parasteatoda tepidariorum. Dashes in the alignment denote gaps introduced to improve the alignment. (DOCX $125 \mathrm{~kb})$
Additional file 7: Alignment of Hth2 proteins from four spider species. Abbreviations: Pp, Pholcus phalangioides; Cs, Cupiennius salei; Ag, Acanthoscurria geniculata; Pt, Parasteatoda tepidariorum. Dashes in the alignment denote gaps introduced to improve the alignment. (DOCX $126 \mathrm{~kb})$

\section{Acknowledgements}

We thank Beate Preitz for help with microscopy. We also thank all members of the department for comments that helped to improve the manuscript.

\section{Funding}

This work has been funded by the Deutsche Forschungsgemeinschaft (grant numbers PR 1109/4-1, PR 1109/6-1 and PR 1109/7-1 to NMP). Additional financial backing has been received from the Göttingen Graduate School for Neurosciences, Biophysics and Molecular Biosciences (GGNB), the Göttingen Center for Molecular Biosciences (GZMB), and the University of Göttingen (GAU). NT is supported by a Christiane-Nüsslein-Volhard-Foundation fellowship and a "Women in Science" Award by L'Oréal Deutschland and the Deutsche UNESCO-Kommission. The funders had no role in study design, data collection and analysis, decision to publish, or preparation of the manuscript.

\section{Availability of data and materials}

DDBJ/ENA/GenBank accession numbers of the sequences used for phylogenetic analysis are listed in Additional file 1. The alignments used for phylogenetic sequence analysis are available in Additional file 2 and Additional file 3. Supporting data is provided in Additional files 5-7.

\section{Authors' contributions}

NT, SK and CS have performed the experiments and documented the data. NMP has conceived the study and drafted the manuscript. NT and NMP designed the experiments, and analysed the data. All authors revised the manuscript for critical intellectual content, read and approved the final manuscript.

Ethics approval and consent to participate

Not applicable.

Consent for publication

Not applicable.

\section{Competing interests}

The authors declare that they have no competing interests.

\section{Publisher's Note}

Springer Nature remains neutral with regard to jurisdictional claims in published maps and institutional affiliations.

\section{Author details \\ ${ }^{1}$ Abteilung für Entwicklungsbiologie, Johann-Friedrich-Blumenbach-Institut für Zoologie und Anthropologie, Georg-August-Universität, Göttingen, Germany. ${ }^{2}$ Göttingen Center for Molecular Biosciences (GZMB), Ernst-Caspari-Haus, Göttingen, Germany. ${ }^{3}$ Present address: Clinic for Cardiology and Pneumology, University Medical Center Göttingen (UMG), Georg-August-University, Göttingen, Germany. ${ }^{4}$ Current address: Georg-August-Universität Göttingen, Johann-Friedrich-Blumenbach-Institut für Zoologie und Anthropologie, Abteilung Zelluläre Neurobiologie, 37077 Göttingen, Germany.}

Received: 20 March 2017 Accepted: 4 July 2017

Published online: 14 July 2017

References

1. Tautz D. The discovery of de novo gene evolution. Perspect Biol Med. 2014:57:149-61.

2. Neme R, Tautz D. Evolution: dynamics of de novo gene emergence. Curr Biol. 2014;24:R238-40.

3. Neme R, Tautz D. Fast turnover of genome transcription across evolutionary time exposes entire non-coding DNA to de novo gene emergence. elife. 2016;5:e09977. 
4. Carrol SB. Evolution at two levels: on genes and form. PLoS Biol. 2005;3:2245

5. Stern DL. Evolutionary developmental biology and the problem of variation. Evolution. 2000;54:1079-91.

6. Flagel LE, Wendel JF. Gene duplication and evolutionary novelty in plants. New Phytol. 2009;183:557-64.

7. Yang Z, Wafula EK, Honaas LA, Zhang H, Das M, Fernandez-Aparicio M, et al. Comparative transcriptome analyses reveal core parasitism genes and suggest gene duplication and repurposing as sources of structural novelty. Mol Biol Evol. 2015;32:767-90.

8. Edger PP, Heidel-Fischer HM, Bekaert M, Rota J, Glöckner G, Platts AE, et al. 2015. The butterfly plant arms-race escalated by gene and genome duplications. Proc Natl Acad Sci U S A. 2015;112:8362-6.

9. Ding Y, Zhao L, Yang S, Jiang Y, Chen Y, Zhao R, et al. A young Drosophila duplicate gene plays essential roles in spermatogenesis by regulating several Y-linked male fertility genes. PLoS Genet. 2010;6:e1001255.

10. Rogers RL, Cridland JM, Shao L, Hu TT, Andolfatto P, Thornton KR. Tandem duplications and the limits of natural selection in Drosophila yakuba and Drosophila simulans. PLoS One. 2015;10:e0132184.

11. Baker RH, Narechania A, Johns PM, Wilkinson GS. Gene duplication, tissuespecific gene expression and sexual conflict in stalk-eyed flies (Diopsidae). Phil Trans R Soc B. 2012;367:2357-75.

12. Rivera AS, Pankey MS, Plachetzki DC, Villacorta C, Syme AE, Serb JM, et al. Gene duplication and the origins of morphological complexity in pancrustacean eyes, a genomic approach. BMC Evol Biol. 2010;10:123.

13. Hasselmann M, Gempe T, Schiøtt M, Nunes-Silva CG, Otte M, Beye M. Evidence for the evolutionary nascence of a novel sex determination pathway in honeybees. Nature. 2008;454:519-22.

14. Arnegard ME, Zwickl DJ, Lu Y, Zakon HH. Old gene duplication facilitates origin and diversification of an innovative communication system-twice. Proc Natl Acad Sci U S A. 2010;107:22172-7.

15. Moriyama Y, Ito F, Takeda H, Yano T, Okabe M, Kuraku S, et al. Evolution of the fish heart by sub/neofunctionalization of an elastin gene. Nat Commun. 2016;7:10397.

16. Clarke TH, Garb JE, Hayashi CY, Arensburger P, Ayoub NA. Spider transcriptomes identify ancient large-scale gene duplication event potentially important in silk gland evolution. Genome Biol Evol. 2015;7:1856-70.

17. Turetzek N, Pechmann M, Schomburg C, Schneider J, Prpic NM. Neofunctionalization of a duplicate dachshund gene underlies the evolution of a novel leg segment in arachnids. Mol Biol Evol. 2016;33:109-21.

18. Ohno S. Evolution by gene duplication. New York, Heidelberg, Berlin: Springer; 1970

19. Force A, Lynch M, Pickett FB, Amores A, Yan Y, Postlethwait J. Preservation of duplicated genes by complementary, degenerative mutations. Genetics. 1999:151:1531-45.

20. Hahn MW. Distinguishing among evolutionary models for the maintenance of gene duplicates. J Hered. 2009;100:605-17.

21. Cao Z, Yu Y, Wu Y, Hao P, Di Z, He Y, et al. The genome of Mesobuthus martensii reveals a unique adaptation model of arthropods. Nat Commun. 2013;4:2602

22. Sanggaard KW, Bechsgaard JS, Fang X, Duan J, Dyrlund TF, Gupta V, et al. Spider genomes provide insight into composition and evolution of venom and silk. Nat Commun. 2014;5:3765.

23. Prpic NM, Janssen R, Wigand B, Klingler M, Damen WGM. Gene expression in spider appendages reveals reversal of exd/hth spatial specificity, altered leg gap gene dynamics, and suggests divergent distal morphogen signaling. Dev Biol. 2003;264:119-40.

24. Pechmann M, Prpic NM. Appendage patterning in the south American bird spider Acanthoscurria geniculata (Araneae: Mygalomorphae). Dev Genes Evol. 2009;219:189-98.

25. Angelini DR, Kaufman TC. Insect appendages and comparative ontogenetics. Dev Biol. 2005;286:57-77.

26. Janssen R, Eriksson BJ, Budd GE, Akam M, Prpic NM. Gene expression patterns in an onychophoran reveal that regionalization predates limb segmentation in pan-arthropods: appendage patterning in onychophorans. Evol Dev. 2010;12:363-72.

27. Mukherjee K, Bürglin TR. Comprehensive analysis of animal TALE homeobox genes: new conserved motifs and cases of accelerated evolution. J Mol Evol. 2007:65:137-53.

28. Bürglin TR. Analysis of TALE superclass homeobox genes (MEIS, PBC, KNOX, Iroquois, TGIF) reveals a novel domain conserved between plants and animals. Nucleic Acids Res. 1997;25:4173-80.
29. Bürglin TR. The PBC domain contains a MEINOX domain: coevolution of Hox and TALE homeobox genes? Dev Genes Evol. 1998;208:113-6.

30. Kurant E, Eytan D, Salzberg A. Mutational analysis of the Drosophila homothorax gene. Genetics. 2001;157:689-98.

31. Pai,CY, Kuo TS, Jaw TJ, Kurant E, Chen CT, Bessarab DA, et al. The Homothorax homeoprotein activates the nuclear localization of another homeoprotein, Extradenticle, and suppresses eye development in Drosophila. Genes Dev 1998;12:435-446.

32. Rieckhof GE, Casares F, Ryoo HD, Abu-Shaar M, Mann RS. Nuclear translocation of Extradenticle requires homothorax, which encodes an Extradenticle-related homeodomain protein. Cell. 1997;91:171-83.

33. Kurant E, Pai CY, Sharf R, Halachmi N, Sun YH, Salzberg A. Dorsotonals/ homothorax, the Drosophila homologue of meis 1, interacts with extradenticle in patterning of the embryonic PNS. Development. 1998;125:1037-48.

34. Ryoo HD, Marty T, Casares F, Affolter M, Mann RS. Regulation of Hox target genes by a DNA bound Homothorax/Hox/Extradenticle complex. Development. 1999:126:5137-48.

35. Ryoo HD, Mann RS. The control of trunk Hox specificity and activity by Extradenticle. Genes Dev. 1999;13:1704-16.

36. Peng HW, Slattery M, Mann RS. Transcription factor choice in the hippo signaling pathway: homothorax and yorkie regulation of the microRNA bantam in the progenitor domain of the Drosophila eye imaginal disc. Genes Dev. 2009:23:2307-19.

37. Bryantsev AL, Duong S, Brunetti TM, Chechenova MB, Lovato TL, Nelson C, et al. Extradenticle and Homothorax control adult muscle fiber identity in Drosophila. Dev Cell. 2012;23:664-73.

38. Casares F, Mann RS. The ground state of the ventral appendage in Drosophila. Science. 2001;293:1477-80.

39. Dong PDS, Dicks JS, Panganiban G. Distal-less and homothorax regulate multiple targets to pattern the Drosophila antenna. Development. 2002; 129:1967-74.

40. Hasegawa E, Kitada Y, Kaido M, Takayama R, Awasaki T, Tabata T, et al. Concentric zones, cell migration and neuronal circuits in the Drosophila visual center. Development. 2011;138:983-93.

41. Wang J, Kean L, Yang J, Allan AK, Davies SA, Herzyk P, et al. Functioninformed transcriptome analysis of Drosophila renal tubule. Genome Biol. 2004;5:R69.

42. Prpic NM, Damen WGM. Expression patterns of leg genes in the mouthparts of the spider Cupiennius salei (Chelicerata: Arachnida). Dev Genes Evol. 2004; 214:296-302.

43. Pechmann M, Khadjeh S, Sprenger F, Prpic NM. Patterning mechanisms and morphological diversity of spider appendages and their importance for spider evolution. Arthropod Struct Dev. 2010;39:453-67.

44. Turetzek N, Prpic NM. Observations on germ band development in the cellar spider Pholcus phalangioides. Dev Genes Evol. 2016;226:413-22.

45. Posnien N, Zeng V, Schwager EE, Pechmann M, Hilbrant M, Keefe JD, et al. A comprehensive reference transcriptome resource for the common house spider Parasteatoda tepidariorum. PLoS One. 2014;9:e104885.

46. Sievers F, Wilm A, Dineen D, Gibson TJ, Karplus K, Li W, et al. Fast, scalable generation of high-quality protein multiple sequence alignments using Clustal omega. Mol Syst Biol. 2011;7:539.

47. Ronquist F, Teslenko M, van der Mark P, Ayres DL, Darling A, Höhna S, et al. MrBayes 3.2: efficient Bayesian phylogenetic inference and model choice across a large model space. Syst Biol. 2012;61:539-42.

48. Kearse M, Moir R, Wilson A, Stones-Havas S, Cheung M, Sturrock S, et al. Geneious basic: an integrated and extendable desktop software platform for the organization and analysis of sequence data. Bioinformatics. 2012;28:1647-9.

49. Schomburg C, Turetzek N, Schacht MI, Schneider J, Kirfel P, Prpic NM, et al. Molecular characterization and embryonic origin of the eyes in the common house spider Parasteatoda tepidariorum. EvoDevo. 2015;6:15.

50. Khadjeh S, Turetzek N, Pechmann M, Schwager EE, Wimmer EA, Damen WGM, et al. Divergent role of the Hox gene Antennapedia in spiders is responsible for the convergent evolution of abdominal limb repression. Proc Natl Acad Sci U S A. 2012;109:4921-6.

51. Mittmann B, Wolff C. Embryonic development and staging of the cobweb spider Parasteatoda tepidariorum C. L. Koch, 1841 (syn: Achaearanea tepidariorum; Araneomorphae; Theridiidae). Dev Genes Evol. 2012;222:189-216

52. Wolff $\mathrm{C}$, Hilbrant M. The embryonic development of the central American wandering spider Cupiennius salei. Front Zool. 2011;8:15. 
53. Nossa CW, Havlak P, Yue JX, LV J, Vincent KY, Brockmann HJ, et al. Joint assembly and genetic mapping of the Atlantic horseshoe crab genome reveals ancient whole genome duplication. GigaScience. 2014;3:9.

54. Kenny NJ, Chan KW, Nong W, Qu Z, Maeso I, Yip HY, et al. Ancestral wholegenome duplication in the marine chelicerate horseshoe crabs. Heredity. 2016;116:190-9.

55. Inoue Y, Mito T, Miyawaki K, Matsushima K, Shinmyo Y, Heanue TA, et al. Correlation of expression patterns of homothorax, dachshund, and Distal-less with the proximodistal segmentation of the cricket leg bud. Mech Dev. 2002;113:141-8.

56. Angelini DR, Kaufman TC. Functional analyses in the hemipteran Oncopeltus fasciatus reveal conserved and derived aspects of appendage patterning in insects. Dev Biol. 2004;271:306-21.

57. Schaeper ND, Wimmer EA, Prpic NM. Appendage patterning in the primitively wingless hexapods Thermobia domestica (Zygentoma: Lepismatidae) and Folsomia candida (Collembola: Isotomidae). Dev Genes Evol. 2013;223:341-50.

58. Prpic NM, Telford MJ. Expression of homothorax and extradenticle mRNA in the legs of the crustacean Parhyale hawaiensis: evidence for a reversal of gene expression regulation in the pancrustacean lineage. Dev Genes Evol. 2008;218:333-9.

59. Mercader N, Leonardo E, Azpiazu N, Serrano A, Morata G, Martínez C, et al. Conserved regulation of proximodistal limb axis development by Meis $1 / \mathrm{H}$ th. Nature. 1999:402:425-9.

60. Corsetti E, Azpiazu N. Functional dissection of the splice variants of the Drosophila gene homothorax (hth). Dev Biol. 2013;384:72-82.

61. Dai M, Wang Y, Fang L, Irwin DM, Zhu T, Zhang J, et al. Differential expression of Meis2, Mab21/2 and Tbx3 during limb development associated with diversification of limb morphology in mammals. PLoS One. 2014:9:e106100.

62. Selden PA, Penney D. Fossil spiders. Biol Rev. 2010;85:171-206.

63. Dunlop JA. Geological history and phylogeny of Chelicerata. Arthropod Struct Dev. 2010;39:124-42.

64. Garrison NL, Rodriguez J, Agnarsson I, Coddington JA, Griswold CE, Hamilton CA, et al. Spider phylogenomics: untangling the spider tree of life. PeerJ. 2016;4:e1719.

\section{Submit your next manuscript to BioMed Central and we will help you at every step:}

- We accept pre-submission inquiries

- Our selector tool helps you to find the most relevant journal

- We provide round the clock customer support

- Convenient online submission

- Thorough peer review

- Inclusion in PubMed and all major indexing services

- Maximum visibility for your research

Submit your manuscript at www.biomedcentral.com/submit 